\title{
EN BUSQUEDA DEL ESTADO DE BIENESTAR A TRAVÉS DE LA FINANCIACION. APORTACIONES DE LA LEGISLACION ESPAÑOLA
}

\author{
IN SEARCH OF THE WELFARE STATE THROUGH FINANCING. CONTRIBUTIONS OF \\ SPANISH LAW
}

\section{Gonzalo Wandosell-Fernández de Bobadilla}

Doctor en Ciencias Económicas. Decano Facultad Ciencias Jurídicas y de la Empresa.

E-mail: gwandosell@ucam.edu

Patricia Blanco-Díez

Doctora en Derecho. Secretaria Académica Grado en Derecho UCAM. E-mail: pblanco@ucam.edu

\section{Carmen-Belén Guillén-Pérez}

Doctoranda Universidad San Antonio de Murcia UCAM (España).

E-mail: belenguillenp@ hotmail.com

Convidados

RESUMEN: La financiación autonómica ha estado en constante evolución en las diferentes etapas a lo largo de estos últimos años. Con este trabajo se observa que la Reforma del año 2009 concluyó con los pilares más sólidos que hasta ahora se habían establecido. Una mayor corresponsabilidad fiscal de la que hasta ahora había en las Comunidades Autónomas, y un mayor logro del Estado de Bienestar como objetivos a conseguir durante este periodo.

Palabras Clave: Comunidad Autónoma. Financiación. Reforma. Corresponsabilidad fiscal. Bienestar social.

ABSTRACT: Autonomous financing has been in constant evolution in the different stages over the last few years. With this work it is observed that the Reform of the year 2009 concluded with the most solid pillars that until now had been established. Greater fiscal co-responsibility than there was until now in the Autonomous Communities, and greater achievement of the Welfare State as objectives to be achieved during this period.

Keywords: Autonomous Community. Financing. Reform. Fiscal co-responsibility. Social welfare.

SUMÁRIO: Introducción; 1. Objetivos de la reforma; 2. Análisis de las etapas de financiación; 3. Planteamiento del nuevo modelo; 4. Finalidad de los acuerdos; Conclusiones; Referencias. 


\section{INTRODUCIÓN}

En estos últimos años, en materia de financiación autonómica, la reforma que más ha influido y que ha ocasionado un mayor impacto económico es sin duda la del año 2009.

Fue a partir del Acuerdo 6/2009 del 15 de Julio, de reforma del sistema de financiación de las Comunidades Autónomas y Ciudades con estatuto propio de autonomía, donde se instauró un sistema que daría lugar a una de las reformas de gran importancia en el sistema de financiación autonómica.

Uno de los factores que determinaron la necesidad de un cambio en el reparto de la distribución financiera entre todas las Comunidades Autónomas fue el aumento de la población de forma no uniforme, particularmente por el motivo de la inmigración, pero el factor determinante fue la aprobación de seis nuevos Estatutos de Autonomía en ese periodo y el problema de la competencia en materia sanitaria de las diferentes Comunidades Autónomas, que dio lugar a un ambicioso proyecto de consolidar una nueva reforma y suplir a la vigente en esos momentos.

El largo proceso de evolución y desarrollo del sistema de financiación autonómica y el debate social y político originado a raíz de los citados cambios en la gestión entre el Estado y las Comunidades Autónomas, concluyó con la creación por parte del Consejo de Política Fiscal y Financiera de las Comunidades Autónomas, en la reunión de 15 de julio de 2009, del Acuerdo 6/2009, en el que se planteó la inmediata reforma del Sistema de Financiación autonómica, dando lugar a una serie de reformas posteriores en los últimos años.

Dicho acuerdo se desarrolla teniendo en cuenta diferentes aspectos de importancia en el marco de las Comunidades Autónomas y de la cesión Estatal de competencias tributarias a las mismas.

El fin principal del proceso de reforma era lograr el denominado "Estado del Bienestar" o, dicho de otra manera, la necesidad de lograr la Equidad entre las Comunidades Autónomas y conseguir la suficiencia financiera necesaria para las competencias autonómicas, en definitiva, intentar conseguir la mayor autonomía y lograr una mayor corresponsabilidad fiscal.

Para conseguir el anhelado Estado de Bienestar, se toma como medio el refuerzo de la asignación de todos los recursos económicos en el nuevo sistema, en base a la política económica de las distintas y diferentes regiones.

Para ello fueron necesarios diferentes recursos creados para reforzar las necesidades mínimas vitales de todos los individuos dentro de cada una de las regiones y encomendando tal fin al Principio de Suficiencia desarrollado en profundidad dentro del concepto de Fondo de Suficiencia Global, cuya finalidad era conseguir una financiación de la mayor parte de las competencias de las Comunidades Autónomas a la vez que se cumplieran algunos objetivos del antiguo modelo financiero.

Para ayudar a tal encomiable fin, se crea el nuevo Fondo de Garantía de Servicios Públicos Fundamentales, con el cual se consigue la equidad deseada por las Comunidades Autónomas menos favorecidas. Así se quiere garantizar que todas las diferentes regiones tengan la misma cantidad de recursos en proporción a cada habitante según la población y necesidades, amparándose en el del artículo 158.1 de la Constitución y del decimoquinto de la Ley Orgánica 8/1980, de Financiación de las Comunidades Autónomas.

Dicho fin no es conseguido en su totalidad, aunque sí existe un aumento de la Equidad en los denominados Fondos de Convergencia creados a través de fondos adicionales del Estado con el fin de mejorar los niveles de vida de los ciudadanos.

Así, en este nuevo sistema de financiación autonómica, algunos principios como el de corresponsabilidad son de mayor aplicación y se produce un mayor aumento de la cesión de tributos a las Comunidades Autónomas.

Se pretendió conseguir la plena colaboración estrecha y recíproca entre las diferentes Administraciones Tributarias y así establecer una cesión con un mayor número de competencias 
tributarias, como delegar en las mismas la revisión de los actos de gestión en vía administrativa.

\section{OBJETIVOS DE LA REFORMA}

El objetivo principal de la citada reforma era estabilizar el sistema, hasta el momento, con multitud de lagunas. Para conseguirlo se instauraron diferentes mecanismos de equilibrio entre las necesidades de una región y el recurso aplicado. Sería necesario considerar cada año las diferentes variables de la región y ver las necesidades reales de las mismas, participando cada Comunidad en el Fondo de Garantía.

La solución al endeudamiento de diferentes Comunidades Autónomas que habían excedido del límite marcado en los presupuestos fue la utilización de métodos de equilibrio que valorasen la liquidación de las Comunidades Autónomas y la posibilidad de aplazar o fraccionar las cantidades pendientes de liquidaciones anteriores.

Para el logro del resto de fines propuestos se creó una serie de recursos que otorgaron mayor aplicación de tributos cedidos por el Estado a las Comunidades Autónomas, realizando transferencias donde se valorasen las necesidades de carácter global y las necesidades reales. Se hizo una valoración de cuáles eran las transferencias que se habían cedido y como se estaban gestionando por las distintas regiones.

La nueva metodología utilizada en esta reforma del 2009 estableció un porcentaje de cesión diferente hasta el ahora vigente. Los diferentes impuestos se elevan a una mayor cesión, destacando entre ellos el Impuesto sobre la Renta de las Personas Físicas del 33\% al 50\%; Productos Intermedios, Alcohol y Bebidas Derivadas, Hidrocarburos y Labores del Tabaco del 40\% al 58\%; el Impuesto sobre el Valor Añadido del 35\% al 50\% y de los Impuestos Especiales de Fabricación sobre la Cerveza, el Vino y Bebidas Fermentadas. Algún sector ve en esta elevación de porcentajes la posible solución a la falta de corresponsabilidad fiscal de la cesión del Impuesto sobre la renta en su totalidad a las Comunidades Autónomas.

Determinados estudios muestran que la cesión total del IRPF proporcionaría a las Comunidades de régimen común una mayor autonomía en ingresos suficientemente amplia y un aumento de corresponsabilidad fiscal. Al cederse en su totalidad, los diferentes espacios fiscales de las regiones el ciudadano de a pie podría diferenciar con claridad de qué servicios dispone y cuales son financiados por su municipio.

Por tanto, se produce un gran cambio, tanto en la forma de la distribución como en la clasificación de las distintas variables y en la determinación de las necesidades reales de los ciudadanos.

Las variables que son utilizadas para clasificar cuales son las necesidades reales de una población pueden ser, entre muchas, las establecidas por los ciudadanos en edad escolar, superficie, insularidad o dispersión, haciendo una clasificación de la población por edades para hacer una calificación del envejecimiento como otra variable más a calcular.

Para llevar a cabo esos objetivos acordes a la nueva reforma de financiación autonómica según el Acuerdo 6/2009 de 15 de julio, intentando conseguir el Estado de Bienestar, se establece una serie de recursos adicionales para mejorar la calidad de vida de los ciudadanos de todas y cada una de las comunidades autónomas, así como mejorar servicios fundamentales como educación sanidad y servicios sociales.

En el tema de la Dependencia, sí se han establecido un numero extenso de prestaciones, de aplicación general entre todos los sectores de la economía y diferentes administraciones.

Hasta entonces, este tipo de recursos eran poco habituales en España, dando lugar a mitigar los déficits de los mismos de las distintas Comunidades Autónomas, aunque esto ha tenido un punto controvertido, y es que ha dado lugar a un aumento de solicitudes de demanda de esos servicios, y en consecuencia a endeudamiento.

La distribución de los recursos se hace según determinados factores, tales como la 
población actual de cada municipio en los últimos años, valorando el término de necesidad según cada habitante y su territorio. Así se consiguió incrementar la suficiencia de las Comunidades y una mayor equiparación de las competencias transferidas a las mismas creando, para llegar al estado de bienestar, el Fondo de Garantía de Servicios Públicos Fundamentales, consiguiendo compatibilizar diferentes principios fundamentales del sistema financiero como la autonomía, la equidad y la corresponsabilidad.

\section{ETAPAS DE FINANCIACION}

El Estado de las autonomías se ha ido formando poco a poco intentando adoptar las nuevas medidas a las necesidades reales, teniendo en cuenta los problemas que iban surgiendo o lo que los gobiernos demandaban, dando lugar a muchos cambios en la gestión.

Muchos de los problemas que se fueron planteando, son debidos a enfoques de tipo político que emborronaban las razones económicas fundamentales. Se observa que a veces los criterios utilizados no han sido los más objetivos posibles, vulnerando algunas veces la equidad.

Han sido varias etapas o periodos los que se han desarrollado en estos 30 años, los cuales deberían ser suficiente para poder cerrar todo el proceso de la financiación autonómica, llegando a la conclusión de que se evidencia que la corresponsabilidad fiscal sigue siendo la asignatura pendiente.

En las diferentes etapas se han ido creados mecanismos para equiparar las diferencias de capacidad fiscal entre las regiones, pero no han sido del todo las suficientes para considerar una aplicación similar.

Tal vez el problema no solo radique en la cesión, si no que su capacidad normativa ha sido limitada y siempre han actuado como gestoras de su propio gasto más que exactoras de los tributos.

A día de hoy, los modelos de financiación de los distintos periodos han visto incrementado su montante total de competencias cedidas, y en muchos casos careciendo de un mayor incentivo para desarrollar y ejercer dichas capacidades. Una de las competencias más recientes y de mayor importancia es la atribución de competencias normativas y a la misma vez el Tribunal Constitucional ha ido clarificando determinados aspectos en referencia a la autonomía tributaria y en cierta medida las Comunidades Autónomas han cogido mayor capacidad normativa y de gestión de tributos.

En este periodo a pesar del auge del cumplimiento de los principios generales del sistema de financiación autonómica, y del notable aumento de la corresponsabilidad fiscal, si se observa que son totalmente insuficientes para establecer un sistema de financiación cedido completamente o en su totalidad a las Comunidades a Autónomas

Se observa una gran diferencia de capacidad fiscal entre el propio Estado y las Comunidades Autónomas, por tanto es largo el camino pendiente para conseguir una mejora en el reparto desigual y un aumento de corresponsabilidad fiscal para aquellas comunidades autónomas que tengan recursos insuficientes.

Hay diferencias significativas en la evolución del sistema de financiación en unas comunidades frente a otras, teniendo un ritmo más lento en aquellas citadas de menores recursos. También es diferente el trato según donde este situada la residencia del obligado a tributar, teniendo unos mayores beneficios fiscales unas que otras y creando un trato discriminatorio que se debería corregir de inmediato.

Todo ello ha dado lugar a diferencias cuantiosas entre las Comunidades Autónomas, siendo mayor si las comparamos con los regímenes forales.

Así desde la reforma del año 2009, queda como tema pendiente, y de una necesaria solución, la escasa responsabilidad cedida y limitación de las Comunidades Autónomas para obtener ingresos, por falta de recursos suficientes.

La capacidad normativa es todavía muy escasa e insuficiente, algunas de las Comunidades 
autónomas, apenas tienen capacidades fiscales respecto a algunos impuestos, ya que estos son compartidos por el Estado y solo participan en su recaudación no en la gestión de este. Por tanto, hay que decir que, con esta reforma, no se aplicaba de manera uniforme el citado principio de solidaridad para lograr un mayor equilibrio entre territorios y entre las diferentes regiones que la forman.

Además, entre todos los principios, es del todo mejorable la aplicación del principio de lealtad institucional entre el Estado y las Comunidades Autónomas, necesario para que el sistema sea estable y duradero.

Si observamos los periodos o quinquenios en donde se ha articulado el sistema de financiación, se empieza en el año 1986 y hasta la fecha ha ido pasando por cinco sistemas o periodos diferentes. Algunos de ellos han querido conseguir un sistema de carácter estable y que fuera duradero en el tiempo, con el fin de adaptarse a las necesidades reales de cada región, considerando la coordinación y la solidaridad como ejes principales para conseguirlo.

Es fundamental para la obtención de dichos objetivos establecer un sistema transparente que pueda adaptarse a los cambios que acontecen, observando las diferentes peculiaridades de cada Comunidad teniendo en cuenta que todos los ciudadanos tengan los mismos derechos, sin crear cierta inestabilidad entre los mismos y encauzando el sistema financiero.

No colaboran a tal fin de la citada transparencia y colaboración los sistemas forales, los cuales no están exentos de críticas, porque se consideran por la gran mayoría como sistemas no participativos y de no colaboración para el mantenimiento del propio Estado, censurando su diferenciación con el resto de las autonomías sin considerar que la aplicación del sistema foral debería ser similar a todas las partes del territorio español.

El criterio que primó para establecer una nueva reforma era intentar conseguir una estabilización general para todas las Comunidades Autónomas, pero el sistema implantado no estaba carente de defectos y erratas que ocasionaban anomalías y asimetrías creando una considerable inestabilidad económica.

Así, una de las características primordiales que caracterizan el modelo del 2009 es su difícil y complejo sistema de nivelación, considerándose poco transparente, igual que los que se habían ido estableciendo en los últimos años.

\section{PLANTEAMIENTO DEL MODELO}

El modelo establecido en esta reforma sienta las bases de cuáles son los cambios acordes a las necesidades reales y al reparto proporcional de ingresos tributarios entre las Comunidades Autónomas, particularmente a nivel de sanidad y de educación.

La idea que se plantea en el mismo es la vocación de permanencia en el tiempo, que sea estable y a la misma vez duradero, un intento por conseguir un sistema con estabilidad en el tiempo y que no tenga la necesidad de acudir de forma reiterada a la arbitrariedad como forma de solucionar problemas que no encuentran una fácil resolución a las injusticias aplicadas en la distribución de los beneficios fiscales en cada Comunidad Autónoma.

No se puede pasar por alto, observando los cambios introducidos en dicha reforma, las diferencias a la hora de financiar las distintas Comunidades Autónomas- Aunque en la mayoría de los casos no se reconozca, el punto de inflexión ha sido diferente, destacando la Comunidad Valenciana como la peor financiada y la Comunidad de Madrid la que obtiene una mayor pérdida de recursos.

La utilización de fórmulas de integración no quiere decir que se vacíe de competencias el Estado ni que las comunidades autónomas adquieran la totalidad de estas, sino que se quiere proceder de una manera proporcional y equilibrada entre los principios de autonomía y de colaboración mutua.

Se adoptan en el modelo unos criterios de integración, instrumentos, normas y protocolos 
de coordinación necesarios para que ambos sistemas, estatales y autonómicos, obtengan un beneficio mutuo y a la vez se mejore el sistema tributario en todo el país.

El planteamiento original de la reforma era reducir las notables diferencias entre las Comunidades, ya que el sistema actual genera muchas desigualdades no justificadas.

Además, el proceso sufrido en los últimos años denominado de "envejecimiento del envejecimiento demográfico" que se puede clasificar con una 'involución poblacional' hace que haya aumentado considerablemente el número de personas dependientes por cada persona en edad de trabajar implicando que nuestra capacidad de financiación de los gastos de tipo social, además del elevado déficit público y la ralentizada devolución de nuestra deuda pública en las próximas décadas, se hará entre un menor número de contribuyentes.

El logro sería conseguir un sistema que evite estos desequilibrios económicos entre las distintas Comunidades Autónomas, evitando las diferencias existentes.

El mayor logro a conseguir por la reforma del 2009 estaba en considerar la aplicación de los impuestos de una forma similar e igualitaria para todas las regiones y que estas puedan ejercer un mayor control sobre los ingresos y sobre los gastos. También se propuso con la reforma obtener, a efectos de impuestos, la concesión para las Comunidades Autónomas del 100\% del impuesto sobre la renta de las personas físicas.

El fin era conseguir una aplicación y una extensión del principio de igualdad, aplicándose a todos los ciudadanos y consolidando que puedan tener acceso a los servicios fundamentales en las mismas condiciones. Pero a pesar del logro conseguido en los servicios públicos fundamentales, queda camino pendiente para que se logre plenamente la deseada igualdad.

Conseguir este logro no está carente de dificultad porque una aplicación absoluta del principio de igualdad es muy complejo dadas las diferencias naturales, geográficas, poblacionales que puedan existir.

Es tal la desigualdad existente que incluso los partidos independentistas catalanes reconocen que deberían aportar una cierta cuota de solidaridad temporal hacia las zonas españolas pobres en un escenario de independencia por el peso de los inmigrantes procedentes de regiones pobres que viven y trabajan en Cataluña a modo de solidaridad interterritorial, a fin de crear mayor armonización entre las Comunidades Autónomas, circunstancia en la que podría haber cierto consenso.

Una de las conclusiones de la reforma del 2009 fue la necesidad de cambios en el reparto de los ingresos tributarios de las Comunidades Autónomas, particularmente en base a los servicios fundamentales de educación y sanidad.

Es importante destacar que la nivelación de la financiación por habitante no garantiza la igualdad en la capacidad de prestación de servicios cuando los territorios que conforman un país presentan diferencias importantes en su estructura demográfica.

El sistema debe establecer un modelo de financiación que tenga mayor equidad y estabilidad a lo largo de los años, porque son muchos las críticas al modelo anterior, de que adolece de defectos.

El sistema aprobado en el año 2009 está incurso en muchos defectos y carencias económicas. Consecuencia de ellos fueron las reuniones donde se realizó la VI Conferencia de Presidentes para intentar lograr unos acuerdos comunes para todas las Comunidades Autónomas.

Cierto es que la capacidad normativa de las Comunidades Autónomas ha ido sustancialmente cambiando, aumentando de una manera considerable. Se ha conseguido que las Comunidades puedan tener decisión en la distribución de sus ingresos y no solo en referencia al volumen, intentando crear nuevas figuras impositivas, las cuales no supongan un coste político.

Dicha reforma establecía el respeto absoluto al principio de solidaridad y nivelación entre las regiones. Para ello se intentó que las Comunidades Autónomas ricas no presentasen tantas diferencias con las que son más pobres y que no solo obtengan beneficios aquellas que tengan mayor capacidad fiscal y aquellas que tengan mayor renta. 
No obstante, el sistema sigue generando a muchas desigualdades que, en la mayoría de los casos no tienen justificación alguna. No existe un reparto justo y equitativo entre todas ellas.

El reparto de la financiación para la distribución entre las Comunidades Autónomas no responde a unos criterios generales o globales entre las mismas. No se tiene en cuenta la población diferente de cada región, ni los valores considerados tales como la edad, el volumen de trabajo y demás variantes a considerar.

\section{FINALIDAD DE LOS ACUERDOS}

La finalidad de todos los acuerdos acontecidos en estos años, son acuerdos consensuados, dando lugar a un modelo estable y duradero.

Uno de los temas pendientes de una solución pronta es considerar que los ingresos no deben tener tantas fluctuaciones en base al ciclo económico, afectando no solo a la modificación financiera a las Comunidades Autónomas como tales, sino a los entes locales de los Ayuntamientos.

Todo el objeto de la reforma financiera siempre ha obedecido en gran medida a la crisis e inestabilidad de las instituciones que se han vivido estos últimos años en el país. La mayor parte de este déficit ha repercutido negativamente en todas las regiones, poniendo en peligro el Estado del Bienestar de las Comunidades Autónomas.

Son varios los motivos que llevo a la reforma del 2009, entre los más destacados se considera que la financiación era escasa y ocasionaba una desigualdad entre las Comunidades Autónomas no solo en el régimen foral sino incluso entre aquellas de régimen común.

Consecuencia de todo ello es la escasez de recursos, no aplicándose el principio de suficiencia en base a las necesidades de estas, pasando por alto las necesidades reales diferentes en cada uno de los territorios.

También es una realidad, sin objeto alguno de discusión, que el anhelado principio de corresponsabilidad está a años luz de cumplirse como tal y el principio de solidaridad entre las regiones es carente de sentido cuando apenas se brinda cooperación entre muchas de ellas.

La reforma establecida en el año 2009 por el Consejo de Política Fiscal y Financiera intentó consolidar ciertos aspectos fundamentales de la financiación autonómica, tales como la ampliación de la cesión tributaria a las Comunidades, aumento de la participación en el IRPF la participación en el IVA, que pasó del 35 al 50\%, así como la participación en los Impuestos Especiales de Fabricación. Para lograr realizar todas las metas planteadas inicialmente en la reforma se creó un complejo sistema de trasferencias de nivelación por medio de una serie de fondos.

Los fondos de Garantía de Servicios Públicos Fundamentales (FGSPF), aseguran que todas las Comunidades puedan ofrecer un nivel similar en la prestación de los servicios públicos fundamentales, como son la sanidad, la educación y los servicios sociales.

El Fondo de Suficiencia Global es similar al anterior Fondo de Suficiencia del modelo que estuvo vigente desde 2002 hasta 2009 y tiene como finalidad contribuir a la financiación de todas las competencias transferidas y asegurarlas.

Pero este sistema se ha caracterizado por la aparición de una serie de problemas viéndose gravemente afectado por la crisis económica iniciada en ese periodo, dando lugar a que los objetivos planteados no pudieran cumplirse en su integridad, tal como se negociaron las condiciones del sistema en el 2009 año que entró en vigor y a partir del cual se inició un periodo en el que el país estaba incurso en una grave crisis nacional.

La diferencia del volumen de recursos entre regiones y la imposibilidad de sostenimiento del principio de suficiencia dio lugar a una fluctuante variación entre regiones. También hubo un aumento considerable entre de los recursos de aquellos tributos cedidos y un detrimento de las transferencias Estatales. Con todo esto no se pudo resolver el problema de la distribución y capacidad normativa, estando muy lejos del fin pretendido. 
No puede obviarse la circunstancia del diferente tratamiento que se aplica entre las Comunidades Autónomas uniprovinciales; al contrario de las Autonomías pluriprovinciales con mayores competencias tributarias.

\section{CONCLUSIONES}

La reforma del año 2009 es sin duda alguna la reforma por excelencia de mayores logros y mayor transformación económica hasta el momento acontecida.

Se creó con vocación de permanencia y con el fin de establecer un sistema estable y duradero, solo modificándose según las circunstancias sin tener que llegar a la modificación total del sistema.

Se ha ido amparándose en un mecanismo de equilibrio que consiguiera armonizar la financiación entre las distintas regiones del territorio español.

Una vez hecho un análisis de esta se observan los diferentes cambios estructurales en el sistema de financiación y es de vital importancia su necesidad y su relevancia en el marco económico.

Para llevar a cabo los objetivos de la reforma se tuvo que atender a las necesidades reales de cada territorio, teniendo en cuenta circunstancias como la demografía, la geografía, el envejecimiento de la población y la dispersión.

El logro de la estabilidad deseada y la permanencia se debió adaptar a los principios constitucionales como principio de autonomía, principio de solidaridad, principio de igualdad, de coordinación y cooperación, principio de suficiencia financiera, para lograr el denominado estado de bienestar, favoreciendo los servicios básicos fundamentales como sanitarios, educativos y sociales.

Es de necesidad y urgencia una reforma de la financiación local, pues se considera que continúa a medio camino, siendo urgente un cambio estructural y de base, porque la Ley 27/2013 LRSAL ha reducido la autonomía local a mínimos, en ocasiones, inapreciables.

Evitaríamos con ello el tema de las duplicidades con las competencias de otras administraciones por tanto sería necesario definir nuevamente la capacidad normativa de las Comunidades Autónomas, debido a la distinta interpretación y en su caso, aplicación de muchas de ellas. definición.

Es preciso valorar aún más la corresponsabilidad fiscal, tema aún pendiente incluso de

Entre las carencias que se observan en dicha reforma se encuentra la existencia cada vez mayor de desigualdades en base al distinto lugar de residencia donde se hallen, tema que se considera debe ser zanjado.

Deberían redistribuirse los recursos tributarios de las regiones, diferenciando los estatales y los de las comunidades autónomas y utilizar criterios uniformes para las mismas sobre la base del concepto de población y de las distintas necesidades de cada una.

Otro punto que trató la reforma fue la distribución del gasto entre las regiones considerándose aquellas que requieren mayor inversión en educación escolar, estudios universitarios, gastos sanitarios de mayores de 70 años, que hasta la fecha no se había desarrollado al respecto.

Dicha reforma establece de manera exhaustiva las clases de tributos, clasificándolos según sea una cesión total o de forma parcial; pero solamente los tributos propios y aquellos cuya cesión es total están gestionados, liquidados o recaudados por las distintas Comunidades.

Algunas Comunidades Autónomas se han ido organizando, estableciendo sus propias Agencias Tributarias en base a la Ley 23/2007 y según lo que establecen sus propios estatutos de autonomía, en cambio, la Agencia Estatal de Administración Tributaria se encarga de los tributos no cedidos, como el Impuesto sobre Sociedades, y aquellos de cesión parcial careciendo las 
Comunidades Autónomas de capacidad normativa sobre el tema.

Lo más destacado es el cumplimiento de los principios de coordinación y cooperación entre el Estado y las Comunidades Autónomas, y el aumento de las posibilidades de mejorar la estimación de la recaudación normativa, capacidad fiscal de las comunidades autónomas en los tributos cedidos de cara a la próxima revisión del sistema de financiación autonómica.

Después de hacer un análisis de la reforma implantada en el año 2009 se llega a la conclusión que el cambio que más urge es sustituir las vigentes reglas de actualización de la recaudación normativa de los tributos cedidos por una regla según la cual dicha recaudación evolucione de forma similar en cada comunidad autónoma. Dicha reforma debería haber llevado un aumento de la recaudación normativa, como ya se hace, por ejemplo, en el IRPF a partir de la información de las liquidaciones tributarias que proporcionen las comunidades autónomas.

Por tanto, uno de los motivos de creación de nuevas normas de financiación autonómica fue por intentar solucionar la disparidad entre regiones, que este periodo no termina de resolver y la capacidad recaudatoria que tiene unas y otras.

La meta que se intenta conseguir después de estos largos años es el cumplimiento del principio de corresponsabilidad fiscal aumentando la capacidad normativa a todas las Comunidades Autónomas.

La larga crisis actual acontecida ha ocasionado una devastadora influencia en la financiación de las Comunidades Autónomas, y lo que originalmente nació para establecer más estabilidad y cesión casi plena de la mayor parte de competencias ha chocado con muchos problemas.

La mayoría de las regiones están exigiendo una mejoría en la suficiencia de recursos de los cuales disponen y a veces no son acordes con las necesidades reales de financiación.

La solución podría verse en un reparto equitativo en lo más ajustado posible de los distintos impuestos y bases tributarias e intentar reducir el endeudamiento que cada vez ha sido mayor, logrando una mayor cesión que se realice efectivamente de la capacidad normativa de las mismas.

Así la reforma de 2009 necesitaba cambios a pesar de ser la que más logros consiguió, si es cierto que se debió de realizar un cálculo de la nivelación y establecer lo que realmente se necesitaba, intentando no sobrepasar los límites que ocasionarían otra vez un aumento del endeudamiento. Consiguiendo con ese estudio un reparto similar a todas las Comunidades Autónomas, pero teniendo en cuenta que las necesidades reales no son iguales para todas las Comunidades Autónomas variando considerablemente entre unas y otras.

Se debió valorar de antemano que la deuda de las Comunidades Autónomas no hubiera llegado a los límites insostenibles que sobrepasaron el mismo principio de estabilidad presupuestaria. Se debió realizar una mejor planificación presupuestaria teniendo en cuenta estas circunstancias de déficit, considerando siempre la coordinación entre ambas administraciones y creando nuevos instrumentos de ayuda para gastos imprevistos.

Pero no se puede pasar por alto que un éxito de la reforma fue la implantación de Fondo de Garantía de Servicios Públicos Fundamentales, aunque si debe revisarse ciertas matizaciones para adecuarlo las necesidades reales de cada una de ellas.

Viendo como se ha desarrollado el sistema de financiación autonómica vigente, la autonomía no se puede considerar como soberanía, con lo que siempre va a ser necesario, al existir en varias Comunidades Autónomas, una coordinación que intente asegurar la aplicación homogénea a todas en condiciones de igualdad, sobre el fundamento de los derechos ciudadanos fundamentales y el establecimiento por igual de los servicios mínimos necesarios.

En materia de coordinación entre las Comunidades Autónomas ha sido el Tribunal Constitucional el que reiteradamente ha establecido el significado del principio de igualdad, considerando iguales en derechos y obligaciones de todos los españoles en cualquier parte del territorio nacional. 
Actualmente se ha puesto de manifiesto que la coordinación entre las distintas Comunidades Autónomas necesita una amplia revisión porque no se ha procedido de manera similar e igualitaria a la hora de redistribuir las competencias en el asunto de la gestión de la pandemia ocurrida el año pasado (2020) del virus Covid 19 y la distribución del Estado Central y las mismas.

\section{REFERENCIAS}

Cantarero Prieto, d. y Fernández Gómez, N. (2005). Suficiencia y corresponsabilidad fiscal regional: Evolución y perspectiva.

Cantarero Prieto, D. (1998). La incidencia de la nueva financiación de las haciendas autonómicas españolas en caso de los servicios de sanidad.

Cantarero Prieto, D. (2016). Financiación autonómica del gasto social: cronicidad y desigualdades.

Castañer Carrasco, J.M., Onrubia Fernández, J. y Paredez Gómez, R., 1998. “Análisis de los efectos recaudatorios y redistributivos de la reforma del IRPF por las comunidades autónomas".

Cubel, M. y Gispert, C. (2010). El Fondo de Compensación Interterritorial: simulaciones de reforma".

De la Fuente, A. (2015). El cálculo de las necesidades de gasto regionales: notas para la discusión.

De la Fuente, A. (2015). El impacto de los factores geográficos sobre las necesidades degasto autonómicas: una aproximación empírica.

De la Fuente, A. (2016). El cálculo de la recaudación normativa de los tributos cedidos tradicionales: una propuesta provisional.

De la Fuente, A. (2017) El cálculo de las necesidades de gasto regionales: notas para la discusión.

Fernández Leicesga, X. y Lago Peñas, S. (2014). “El modelo de financiación autonómica desde la perspectiva de los gobiernos autonómicos.

Fernández Llera, R. (2016). El encaje entre financiación autonómica y estabilidad presupuestaria en torno a la coordinación y la planificación.

Garcimartín, C. Díaz de Sarralde S. y Ruíz-Huerta, J. (2013). El actual modelo de Financiación Autonómica: efectos sobre la convergencia.

González González, A. y Álvarez González, S. (2011). Panorama actual de los tributos propios de las Comunidades Autónomas.

González González, A.I. y Álvarez González, S. ( 2015). Panorama actual de los tributos propios de las Comunidades Autónomas españolas.

Granell, F. (2016). Algunas ideas sobre la reforma del sistema de financiación autonómica. 
Hierro Lecio, L.A. Atienza Montero, P. (2016) .El efecto estabilizador de la financiación autonómica de régimen común.

López Laborda, L., (2016) La medición de la capacidad fiscal de las comunidades autónomas: algunas alternativas.

Martínez-Vázquez, J. (2016). El papel de los impuestos propios en la financiación autonómica: Lecciones de la experiencia internacional.

Onrubia J. (2016). Financiación autonómica y administración tributaria: Una propuesta de modelo integrado de gestión.

Pedraja Chaparro, F. y Ultrilla de la HOz, A. (2011). "Autonomía y equidad en el nuevo sistema de financiación autonómica.

Pérez Francesch, J.L. (2006). El estado de las autonomías a debate. Una reflexión desde Cataluña.

Pérez, A. (2016). Problemas básicos y perspectivas de futuro de la financiación autonómica y local.

Pérez, P., Beneyto, R., Pérez, J.A., Fernández, J.I., Peiró, V. y Cucarella, V. (2013). Criterios y propuestas para un nuevo sistema de financiación autonómica.

Sánchez Fernández, J. (2007). Financiación autonómica y población.

Soler Marco, V. (2016). Financiación y modelo económico. Valencia.

Zabalza, A. (2016). Equidad y responsabilidad en la financiación autonómica.

Zoco Zabala, C. (2015). Articulación autonómica de las competencias locales tras la LRSAL: hacia una autonomía local como derecho.

Zubiri, I. (2016). El Sistema de Financiación de las CC.AA.: Problemas y Propuestas de Solución.

\section{APÉNDICE JURISPRUDENCIAL}

STC 37/1981, de 16 de noviembre, Fundamento Jurídico Segundo. 